\title{
Usefulness of 3D-PISA as compared to guideline endorsed parameters for mitral regurgitation quantification
}

\author{
Frank P. Schmidt • Theresa Gniewosz • \\ Alexander Jabs • Thomas Münzel • Ulrich Hink • \\ Patrizio Lancellotti • Ralph-Stephan von Bardeleben
}

Received: 23 February 2014 / Accepted: 9 July 2014

(C) Springer Science+Business Media Dordrecht 2014

\begin{abstract}
This study was intended to evaluate the diagnostic value of three dimensional proximal isovelocity surface area (3D PISA) derived effective regurgitant orifice area (EROA) and the accuracy of automatic 3D PISA detection in a population resembling clinical practice. Quantification of mitral regurgitation (MR) remains challenging and 3D PISA EROA is a novel diagnostic tool with promising results. However its' usefulness compared to guideline endorsed parameters has not been shown. In 93 consecutive patients examined in routine practice conventional parameters and 3D-datasets for offline 3D PISA evaluation were recorded. EROA was determined from the largest (peak) PISA and also averaged over systole for meanEROA. Results of 3D PISA calculation were compared with a combination of expert grading by two examiners and two scores for MR grading. In receiver operator characteristic-analysis the meanEROA as determined by 3D PISA had the best diagnostic value $(\mathrm{AUC}=0.907$ CI $0.832-0.983)$ as compared to peakEROA (AUC 0.840 CI 0.739-0.941), vena contracta width (AUC 0.831 CI 0.745-0.918) and 2D PISA (AUC 0.747 CI $0.644-0.850$ ). A meanEROA of $0.15 \mathrm{~cm}^{2}$ had a sensitivity
\end{abstract}

Electronic supplementary material The online version of this article (doi:10.1007/s10554-014-0496-7) contains supplementary material, which is available to authorized users.

F. P. Schmidt $(\bowtie) \cdot$ T. Gniewosz · A. Jabs · T. Münzel ·

U. Hink - R.-S. von Bardeleben

Department of Medicine II, University Medical Center of

Johannes Gutenberg-University Mainz, Langenbeckstr. 1,

55131 Mainz, Germany

e-mail: frank.schmidt@unimedizin-mainz.de

P. Lancellotti

GIGA Cardiovascular Sciences, Heart Valve Clinic, CHU Sart

Tilman, University of Liège Hospital, Liège, Belgium of $88.2 \%$ and a specificity of $81.4 \%$ for distinguishing severe from non-severe MR. Semiautomatic 3D PISA detection correlated very well with manually corrected values $(r=0.955)$. Semiautomatic 3D PISA measurement is feasible in a clinical population and has better diagnostic value compared to 2D PISA. Calculation of mean EROA throughout systole further improves diagnostic value compared to conventional parameters.

Keywords Mitral regurgitation - 3D echocardiography . 3D PISA

$\begin{array}{ll}\text { Abbreviations } \\ \text { 2D } & \text { Two dimensional } \\ \text { 3D } & \text { Three dimensional } \\ \text { AR } & \text { Aortic regurgitation } \\ \text { AUC } & \text { Area under the curve } \\ \text { BMI } & \text { Body mass index } \\ \text { bpm } & \text { Heatbeats per minute } \\ \text { CAD } & \text { Coronary artery disease } \\ \text { CKD } & \text { Chronic kidney disease } \\ \text { COPD } & \text { Chronic obstructive pulmonary disease } \\ \text { E/E' } & \text { Early mitral inflow velocity/early mitral annulus } \\ & \text { velocity } \\ \text { EDV } & \text { Enddiastolic volume } \\ \text { EROA } & \text { Effective regurgitation orifice area } \\ \text { ICC } & \text { Intraclass correlation coefficient } \\ \text { LA } & \text { Left atrium } \\ \text { LVEF } & \text { Eleft ventricular jection fraction } \\ \text { MR } & \text { Mitral regurgitation } \\ \text { NYHA } & \text { New York Heart Association classification } \\ \text { PISA } & \text { Proximal isovelocity surface area } \\ \text { PMR } & \text { Primary mitral regurgitation } \\ \text { ROC } & \text { Receiver operator characteristic } \\ \text { RVol } & \text { Regurgitation volume } \\ \end{array}$

\section{Abbreviations}

Two dimensional

Three dimensional

Aortic regurgitation

AUC Area under the curve

BMI Body mass index

bpm Heatbeats per minute

CAD Coronary artery disease

CKD Chronic kidney disease

COPD Chronic obstructive pulmonary disease

EDV Enddiastolic volume

EROA Effective regurgitation orifice area

ICC Intraclass correlation coefficient

LA Left atrium

LVEF Eleft ventricular jection fraction

MR Mitral regurgitation

NYHA New York Heart Association classification

PISA Proximal isovelocity surface area

ROC Receiver operator characteristic

RVol Regurgitation volume 
SD Standard deviation

SMR Secondary mitral regurgitation

SPAP Systolic pulmonary artery pressure

VC Vena contracta width

VCA Vena contracta area

\section{Introduction}

A more accurate quantification of mitral regurgitation (MR) is of great clinical importance for identifying patients suitable for surgical or interventional valve procedures. However, after decades of research still no true gold standard for use in clinical practice exists. Grading of MR takes into account many parameters and requires expert knowledge and experience. Advances in ultrasound technology, especially the increasing availability and technical sophistication of 3D-echocardiography should therefore be used to improve determination of regurgitation severity. One of those new and promising methods is three dimensional (3D) evaluation of the proximal isovelocity surface area (PISA). The conventional 2D-PISA has been shown to correlate well with angiography [1] and thresholds separating severe from non-severe MR have been obtained and included into clinical guidelines [2-4]. However limitations and pitfalls have long been recognized [5, 6]. Compared to 2D-PISA, 3D-PISA has theoretical advantages, especially with regard to its superior applicability in noncircular orifices. It has been shown that 2D-PISA underestimates regurgitation in non-circular regurgitation orifices [7, 8] and correlates better with vena contracta area (VCA) in circular orifices [9] [10]. Despite these appealing advantages and encouraging results from early studies [1115], 3D-PISA was slow to reach clinical practice, initially because of poor temporal resolution and also analysis remained difficult for some time. Recently an automated segmentation of the 3D-isovelocity surface area was shown to produce good results to correctly classify MR severity categories [16] in vitro and in single patients [17]. The 3DPISA method has subsequently been compared with another 3D color Doppler method, the VCA with both measures correlating very well in a small number of patients [18]. In addition 3D-PISA has been integrated over the cardiac cycle with its known phasic variations in regurgitation and the integrated measure compared to results from MRI [19], again showing good results. Judging from these evaluations of the method, 3D-PISA appears to be very promising for future grading of regurgitation. However, these results were either obtained in vitro or in a very selected group of patients with almost perfect image quality and no complicating factors such as atrial fibrillation or concomitant regurgitation of other valves. Although appealing these single comparators (MRI, VCA) have not been sufficiently validated in terms of clinical correlation and reliance on single parameters is rightfully discouraged by current guidelines.

In order to incorporate the 3D-PISA into clinical practice its diagnostic utility in routine echocardiography has therefore to be evaluated against established methods and current guideline based grading. While 2D methods like vena contracta width and 2D-PISA are less accurate in secondary (functional) mitral regurgitation (SMR), 3DPISA should theoretically perform well in both primary and secondary valvular regurgitation. Since PISA-diameter has been shown to vary substantially during systole [20, 21], we intended to include an analysis accounting for this dynamic changes and verify diagnostic accuracy for averaged 3D-PISA compared to single point determination.

We examined routine cases with MR of all etiologies and performed complete transthoracic exams including datasets with 3D-PISA acquisition. Diagnostic accuracy and classification of MR with 3D-PISA based EROA and also mean EROA was to be tested against other measures, scores and expert judgment.

\section{Methods}

Consecutive patients referred for transthoracic echocardiography to our institution, a large tertiary echocardiography lab, which were found to have MR and had a sufficient apical echo window where included in this study. Patients with greater than moderate aortic valve regurgitation or with more than mild aortic stenosis were excluded. No other exclusion criteria were used for patient selection.

2D images and 3D datasets were acquired using a ACUSON SC2000 (Siemens Medical, Erlangen, Germany) ultrasound machine with a $4.5 \mathrm{MHz} 2 \mathrm{D}$ transducer (4V1) and a $2.5-\mathrm{MHz}$ single-beat 3D-Volume transducer (4Z1c Siemens Medical Solutions, USA). Image quality had to be satisfactory for grading regurgitation and acquisition of 3D-PISA images. For the 3D volume acquisition from the apical four chamber view, the "S1" preset of the machine (emphasis on spacial resolution) was used and volume size, gain, dynamic range, Nyquist limit and volume position were adjusted to ensure optimal acquisition of the complete PISA shell. At least three separate color-volume data sets with two or more consecutive heart beats were stored for later analysis. The average volume rate of the acquired 3Ddatasets was $16.7 \pm 2.1$ volumes per second (vps).

Data was analyzed offline on a separate workstation with Siemens Workplace software (Siemens, Mountain View, USA). 2D data analysis and measurements were performed according to current guidelines [3], including 


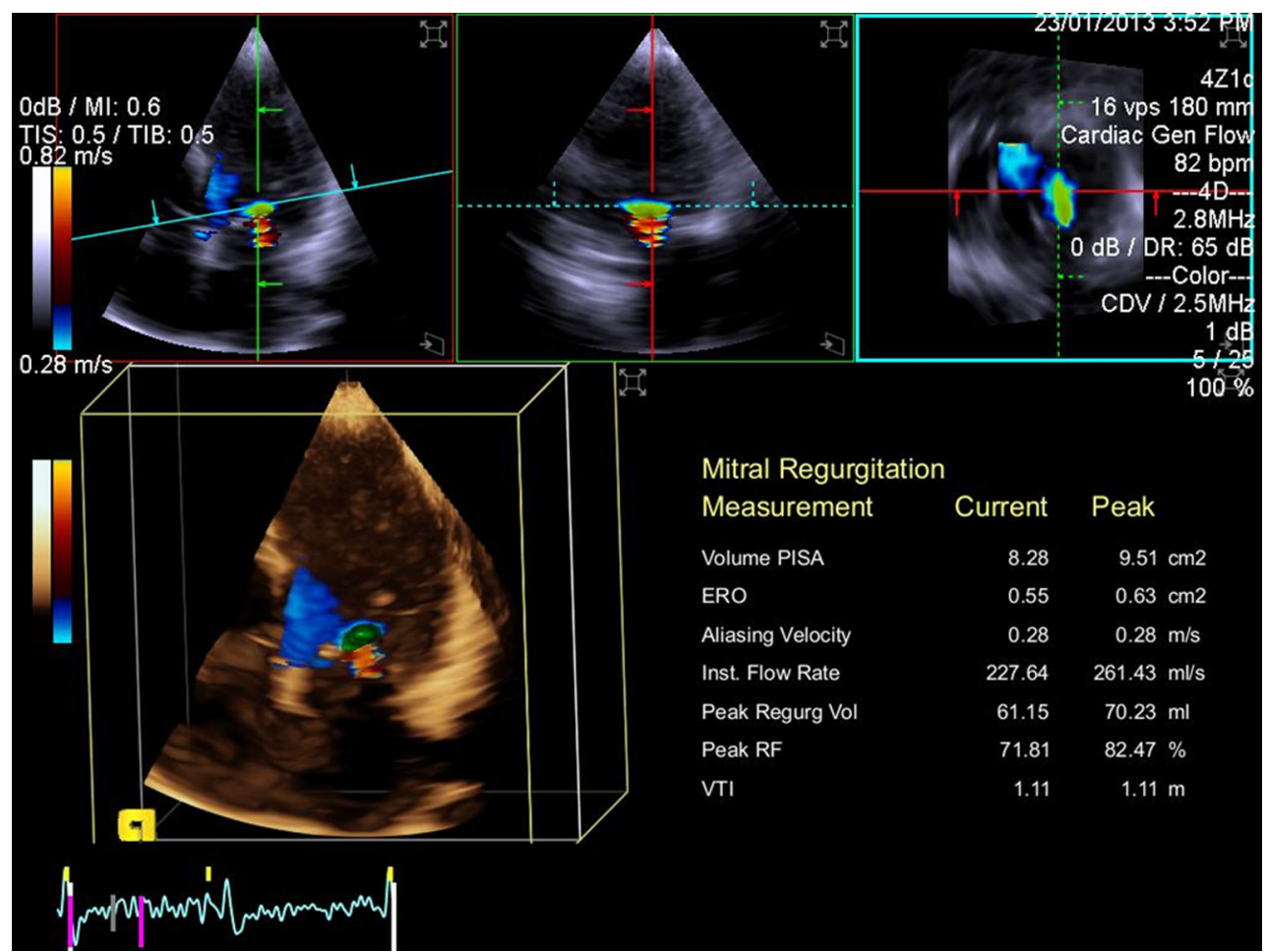

Fig. 1 Semi-automated 3D-PISA evaluation. Example of 3D-PISA evaluation with repetitive calculation of EROA from the 3D-PISA shell (green). The shell is generated by a semi-automated algorithm and can be examined in all three dimensions and edited manually if necessary. Current and peak values are displayed in this case of secondary MR with elongated PISA

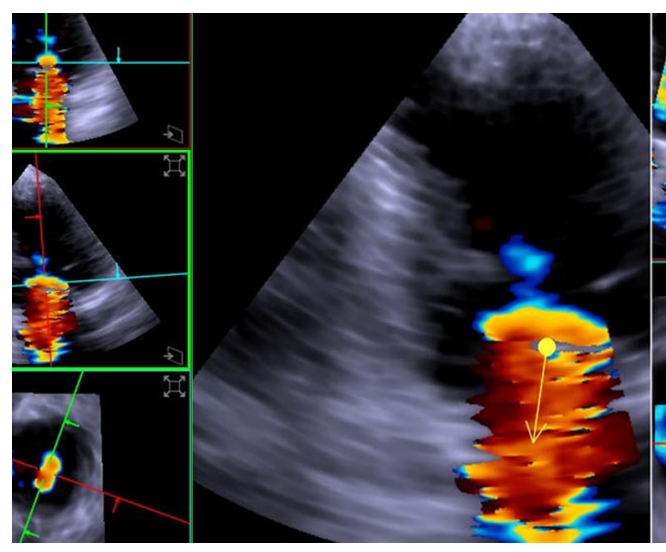

Fig. 2 Semiautomatic 3D-PISA detection algorithm. After image acquisition of 3D volume data, 3D-PISA analysis is performed with the eSiePISA algorithm. The examiner indicates the location of the PISA by placing a dot a the base and pointing an arrow for indicating

averaging of measurements in case of atrial fibrillation. 3D datasets were evaluated taking into account image quality and heart rhythm with the most representative volume being selected for 3D-PISA analysis. EROA was determined for every systolic volume (as in frame) with identifiable MR and PISA-shell. 3D-PISA calculation was done offline with the use of a semiautomated algorithm (Siemens eSIE PISA) (Figs. 1, 2) and manually corrected using the

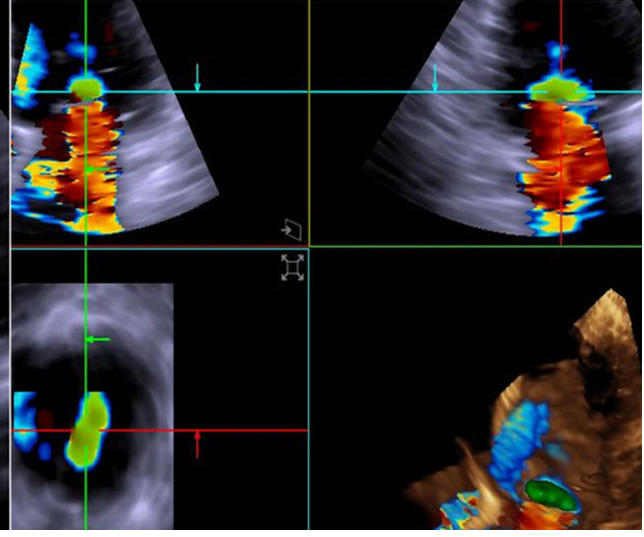

the jet direction. After this 2-click procedure the 3D-PISA is detected automatically and the detected surface is indicated in green and can be visually checked for the quality of the detection

same software. Manual correction to the detected PISA shell was performed to get a best fit to the color Doppler PISA volume at the appropriate Nyquist limit (Fig. 3). If necessary the 3D-PISA was scrolled through and edited in two orthogonal planes. The maximum regurgitation orifice area (peakEROA) and the mean regurgitant orifice area (meanEROA) were determined using the maximum regurgitation jet velocity. The mean EROA was calculated 
Fig. 3 Manual correction of 3D PISA. Example for the manual changes that can be made to the automatically detected PISA shell. On the left, the detected 3D-PISA (green) does not include the complete shell, as can be seen by the yellow residual. The right image demonstrates a manual correction result after painting the yellow areas green up to the border of color change at the respective Nyquist limit

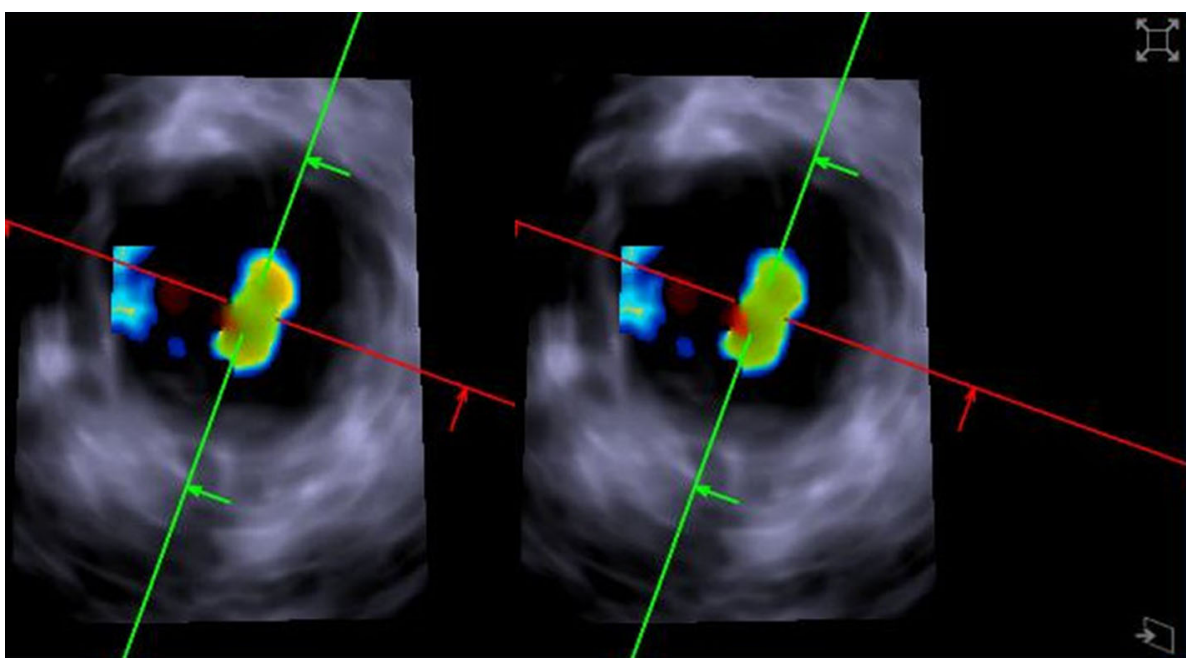

by dividing the sum of all EROAs (calculated from consecutive 3D-PISA shells; Fig. 2) by the number of systolic volume frames.

\section{Grading of mitral regurgitation severity}

MR severity was graded by the physician performing the echocardiographic exam (taken from the written report) and again separately after data collection by a single expert echocardiographer unaware of the original report. Both readers were Level 3 trained [22] and grading was done per standard of current EACVI recommendations [3]. Neither the initial examiner nor the second reader had results from 3D-PISA analysis at the time of grading MR. Jet area was measured in the standard four chamber view, vena contracta width in the four chamber or long axis view.

In addition we determined MR severity with the use of the scoring system proposed by Buck et al. [23] and the MR Index introduced by Thomas et al. [24]. As standard for comparison we calculated a meta-score integrating the two gradings of echocardiographers and both scoring systems $\quad$ (meta-score $=($ examiner $1+$ examiner $2+$ (BuckScore $)^{*} 0.4+($ MR Index $\left.\left.) * 2\right) / 4\right)$. As a cutoff for significant MR we used a meta-score of $>3.5$. This cutoff was chosen prior to analysis to give equal weight to all components of the score according to published cutoffs for differentiating moderate from moderate-to-severe MR.

Clinical data was obtained retrospectively from electronic patient records. Echocardiographic data was remeasured offline separately from the original exam.

Data was scrutinized for plausibility and outliers. Unusual values were then rechecked from the original data.
Table 1 Patient characteristics

\begin{tabular}{|c|c|c|}
\hline Age & $71.2 \pm 12.01$ & years \\
\hline Female sex & 38.7 & $\%$ \\
\hline BMI & $26.1 \pm 4.0$ & $\mathrm{~kg} / \mathrm{m}^{2}$ \\
\hline \multicolumn{3}{|l|}{ Rhythm } \\
\hline Sinus & 52.7 & $\%$ \\
\hline $\mathrm{AF}$ & 37.6 & $\%$ \\
\hline \multicolumn{3}{|l|}{ NYHA class } \\
\hline $\mathrm{I} / \mathrm{II}$ & 48.2 & $\%$ \\
\hline III/IV & 51.8 & $\%$ \\
\hline CAD & 79.6 & $\%$ \\
\hline Hypertension & 81.7 & $\%$ \\
\hline CKD & 51.1 & $\%$ \\
\hline COPD & 14.1 & $\%$ \\
\hline Diabetes & 23.6 & $\%$ \\
\hline Secondary MR & 80.4 & $\%$ \\
\hline \multicolumn{3}{|l|}{ Mitral regurgitation } \\
\hline Mild & 6.5 & $\%$ \\
\hline Moderate & 23.7 & $\%$ \\
\hline Moderate to severe & 26.9 & $\%$ \\
\hline Severe & 43.0 & $\%$ \\
\hline $\mathrm{L} / \mathrm{S}>1.5$ & 60.2 & $\%$ \\
\hline RVol 2D & $33.3 \pm 21.9$ & $\mathrm{ml}$ \\
\hline RVol 3D & $47.6 \pm 27.6$ & $\mathrm{ml}$ \\
\hline Aortic regurgitation & 50.6 & $\%$ \\
\hline $\operatorname{AR} \%>\mathrm{I}^{\circ}$ & 7.6 & $\%$ \\
\hline LVEF & $34.7 \pm 12.9$ & $\%$ \\
\hline EDV & $201.5 \pm 79.8$ & $\mathrm{ml}$ \\
\hline LA area & $28.95 \pm 8.73$ & $\mathrm{~cm}^{2}$ \\
\hline sPAP (TR) & $39.33 \pm 10.26$ & $\mathrm{mmHg}$ \\
\hline $\mathrm{E} / \mathrm{e}^{\prime}$ & $12.0 \pm 7.2$ & \\
\hline
\end{tabular}




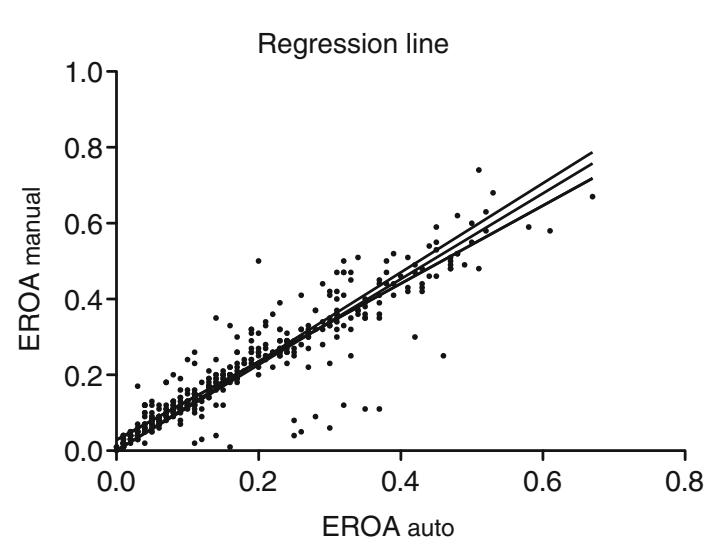

Fig. 4 Correlation between manually corrected and automatically detected 3D-PISA. Linear regression between automatically and manually traced PISA shells with 436 of 465 PISA shells analyzed. Pearson's $r=0.931$

Outliers in linear regression were determined in SPSS by calculating cook's distance and excluding cases with values $>0.007$. All outliers were more than two SD from the mean residual. To calculate indices from variables we used formulas in Excel data sheets (Excel for Mac 2011, Microsoft, USA). For statistical analysis we used SPSS Statistics version 20.0 (IBM Corp., USA) and Prism 5 for MacOS (GraphPad Software, La Jolla, USA). Continuous variable are expressed as mean $\pm \mathrm{SD}$, categorical variables are given in absolute numbers or percentage of. $p$ values $<0.05$ were considered significant.

\section{Results}

A total of 93 (57 male, 36 female) patients with MR (17 primary, 76 secondary MR) were available for analysis. Of those six were judged to have mild, 22 moderate, 25 moderate to severe and 40 to have severe MR by the initial examiner. 47 patients had some degree of aortic regurgitation, mostly mild AR $(85 \%) .49$ patients were in sinus rhythm during the exam, 35 in atrial fibrillation and 9 patients had paced ventricular rhythm. $29.2 \%$ of patients had regurgitation jets with two jet components, however only on confluent PISA shell. Patients with two or more completely separate jets were not included in the study. $91.4 \%$ of patients had a jet impinging on at least one atrial wall. The average heart rate was $73.8 \pm 19.8$ beats per minute (bpm) with $90 \%$ of patients having less than $100 \mathrm{bpm}$. Further details about the study population are given in Table 1 .

\section{D-PISA detection}

3D-PISA shells demonstrated on average a moderate ovaloid shape with a mean L/S ratio (larger diameter/smaller

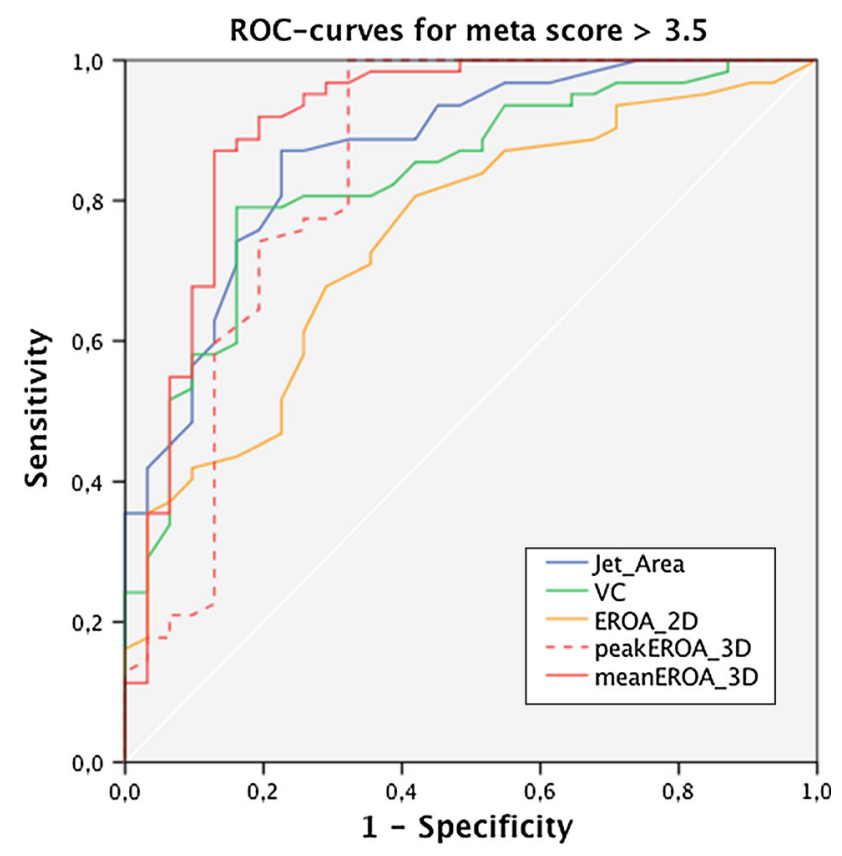

Fig. 5 ROC curves against meta score at 3.5 cutoff. Receiver operator characteristics curve for different quantitative measures of MR severity using a cutoff of our meta-score of 3.5, meaning more than moderate MR. Red meanEROA 3D-PISA derived, interrupted red peakEROA 3D-PISA derived, orange EROA 2D-PISA derived, green $\mathrm{VC}$, blue jet area

diameter) of $1.68 \pm 0.61$. The distribution showed one maximum around $\mathrm{L} / \mathrm{S}=1$ and another one around $\mathrm{L} / \mathrm{S}=2$. The median Nyquist limit used was $0.28 \mathrm{~m} / \mathrm{s}$. For calculation of meanEROA from 3D-PISA an average of $6.7 \pm 1.4$ systolic frames were analyzed. For comparison of semiautomically determined and manually corrected 3D-PISA values, 465 data sets were available for analysis. After outlier elimination as described above, 434 data sets remained for analysis. The semiautomatically determined 3D-PISA shell had a mean surface of $2.799 \pm 2.134 \mathrm{~cm}^{3}$ compared to $3.339 \pm 2.287 \mathrm{~cm}^{3}$ for manually corrected PISA shells. The resulting EROA for semiautomated detection was $0.166 \pm 0.138 \mathrm{~cm}^{2}$ versus $0.197 \pm 0.152 \mathrm{~cm}^{2}$ for manually corrected detection. Correlating semiautomated and manual corrected values, Pearson's $r$ was $r=0.909(p<0.001)$ for 3D-PISA volume and $\mathrm{r}=0.931(p<0.001)$ for EROA determination (Fig. 4).

Analysis using automated outlier detection resulted in the exclusion of 45 outliers or $9.7 \%$ of results. The best-fit line was calculated as $\mathrm{y}=1.073 * \mathrm{x}+0.026$ with $\mathrm{y}$ being manual corrected EROA and $\mathrm{x}$ being automated PISA detection. This model had an excellent fit with $\mathrm{r}^{2}=0.955$.

\section{Mitral regurgitation assessment}

The intraclass correlation coefficient for the readers grading the MR was ICC $=0.869(0.801-0.914)$ for absolute 


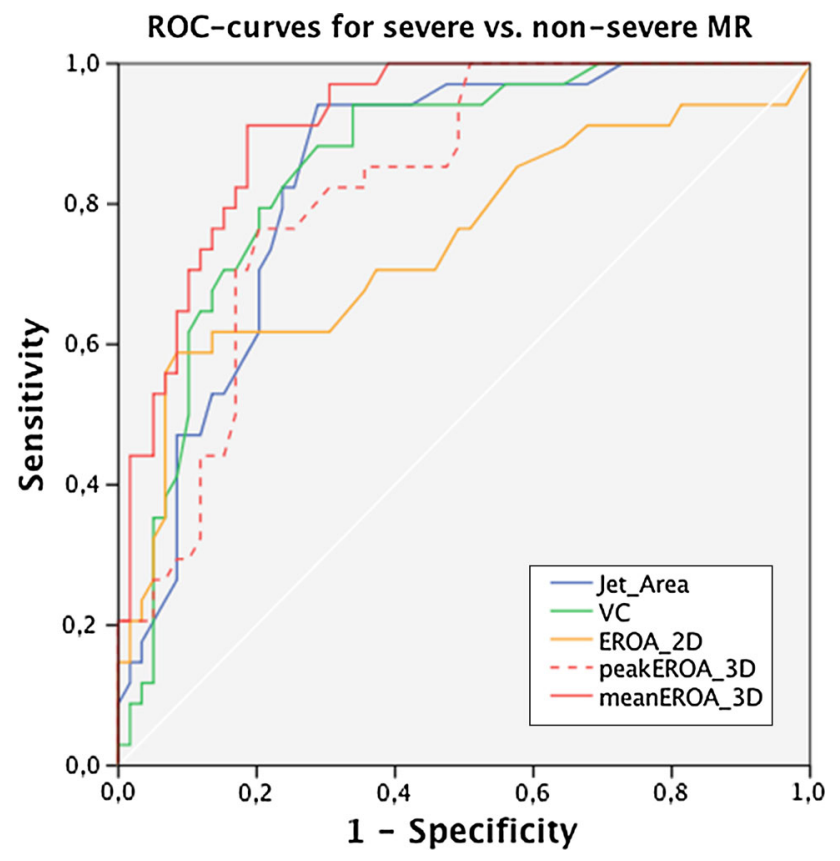

Fig. 6 ROC curves for expert grading. Receiver operator characteristics curve for different quantitative measures of MR severity using expert grading severe versus non-severe. Red meanEROA 3D-PISA derived, interrupted red peakEROA 3D-PISA derived, orange EROA 2D-PISA derived, green $\mathrm{VC}$, blue jet area

agreement. The average modified MR Index was 1.79 $( \pm 0.41)$ and the average point score according to Buck et al. was $10.21( \pm 1.71)$.

The average EROA and Regurgitation volume (RVol) were significantly larger for 3D-PISA (EROA $0.33 \pm$ $0.19 \mathrm{~cm}^{2}$ and Rvol $47.6 \pm 27.6 \mathrm{ml}$ ) compared to 2D-PISA (EROA $0.21 \pm 0.13 \mathrm{~cm}^{2}$ and $\mathrm{RVol} 33.3 \pm 21.9 \mathrm{ml}$; $p<0.001)$. There was no significant difference between PMR and SMR in this respect.

In a receiver operator characteristics curve analysis (ROC) using the meta score described above, the mean 3DEROA as determined with 3D-PISA performed best (AUC $=0.907$ CI 0.832-0.983) compared with the peak 3D-EROA (AUC 0.840 CI 0.739-0.941), EROA calculated from 2D-PISA (AUC 0.747 CI 0.644-0.850), vena contracta width (AUC $0.831 \mathrm{CI} 0.745-0.918$ ) or jet area (0.869 CI 0.793-0.945) (Fig. 5).

Compared to the MR grading of the second experienced reader, mean3D-EROA again performed better (AUC 0.914 CI $0.859-0.969)$ in ROC analysis than the other parameters for distinguishing severe from non-severe MR (peak 3D-EROA AUC $=0.822$ CI $0.739-0.906 ; 2 \mathrm{D}-$ $\mathrm{EROA}$ AUC $=0.747$ CI $0.636-0.858 ; \mathrm{VC}$ AUC $=0.855$ CI 0.778-0.933; Jet area $\mathrm{AUC}=0.842$ CI 0.842-0.922) (Fig. 6).

A meanEROA of $0.15 \mathrm{~cm}^{2}$ had a sensitivity of $88.2 \%$ and a specificity of $81.4 \%$ for distinguishing severe from non-severe MR. For peakEROA a cutoff of $0.36 \mathrm{~cm}^{2}$ had a sensitivity of $76.5 \%$ and a specificity of $79.7 \%$ for distinguishing severe from non-severe MR.

When looking at spherical versus non-spherical (L/S ration $>1.5$ ) PISA shells, meanEROA was better for more spherical PISA (AUC 0.965 vs. 0.878) a difference that was even more pronounced for peakEROA (AUC 0.914 vs. 0.767). The same relationship was seen for 2D-PISA, but at lower AUC values (AUC $=0.822$ for $\mathrm{L} / \mathrm{S} \leq 1.5$ vs. AUC $=0.706)$ Separate evaluation of severe PMR and SMR showed larger AUC for meanEROA in PMR (0.948 vs. 0.904) but these results are based on rather small number of PMR in the study population. No significant gender differences were found.

\section{Discussion}

The results of this study demonstrate the utility and diagnostic value of an averaged effective orifice area as determined by the 3D-PISA method. In contrast to earlier studies we examined a population of patients that more closely resembles the patients encountered in daily practice. Patients with rhythm disturbances such as atrial fibrillation and ventricular pacing were included just as were patients with less than optimal acoustic windows. For the first time we report the performance of a semiautomated algorithm in comparison to manual correction in the clinical setting. Except for obvious aberrations, the algorithm produces numerically smaller values with very good correlation to manual tracing of the PISA.

When new diagnostic methods are introduced, we would like to establish their value in comparison with the current clinical standard. In practice it is less important how well the numbers of two methods (like EROA and VCA) match, than if the new quantitative measure is better for clinical decision making than those we currently use.

We were able to demonstrate that the meanEROA is superior to other quantitative measures of regurgitation severity in diverse groups of patients.

Other authors have chosen to use an averaged regurgitation volume for their 3-D integrated PISA [19]. Both approaches appear justified since basically the same measurements are used for calculation of integrated RVol and meanEROA. 3D-PISA derived meanEROA demonstrated its diagnostic performance with better AUC than any other measure. Contrary to earlier studies [1] we found jet area and vena contracta width to be superior to 2D-PISA in our cohort, probably due to the predominance of SMR.

Since no gold standard exists for grading of MR severity, new methods are often tested against either expert opinion or a single comparator method like MRI. While legitimate, this practice has obvious limitations with regard 
to objectivity and generalizability. In order to make our comparator more objective while also using an integrated approach, we decided to combine the results of two readers (using guideline based integrative grading) and established scores using multiple quantitative parameters. This metascore is a novel way for comparison of different parameters, especially in patients where other methods may not be applicable e.g. due to atrial fibrillation, aortic regurgitation or the presence of pacemaker leads.

Whereas previous studies used values as calculated by the semiautomated analysis tool and reported good results, we also used the possibility to make manual corrections to the 3D-PISA surface detection. Although differences are negligible in many cases, it is necessary in some cases with problematic automated detection. The availability of a manual correction to PISA detection may be of considerable usefulness in a population with less than optimal conditions. While there was a very good correlation of values for over $90 \%$ of cases, some outliers will need manual editing. From our experience we would recommend to use the semiautomated analysis tool, check the PISA detection visually and make corrections only for relevant discordances. While corrections may often not be necessary, checking the results of automated detection should be mandatory.

Evaluation of PISA with the use of one-beat real-time 3D-echocardiography has many theoretical and also practical advantages compared to other methods. The PISA shell is closer to the transducer in TTE and less effected by valvular calcifications or other sources of ultrasound dropout than methods that assess flow on the more distal atrial side. Also 3D-PISA does not rely on determining a certain cut plane for measurement (in contrast to VCA). In our own experience this reduces interobserver variability. 3D-PISA should be superior to the conventional 2D-PISA especially in cases were the assumption of the PISA being more or less spherical does not hold true. This is the case in most patients with functional MR. 3D-PISA can be performed non-invasively during routine transthoracic exam with relatively fast data acquisition due to the one-beat technology. Interestingly, we found a better diagnostic performance in round rather than elongated PISA shells not only for 2D-PISA but also for 3D-PISA. This finding suggests that recording the PISA shell in three dimensions does not completely eliminate all reasons why PISA works better for round orifices (e.g. Doppler angulation and velocity distribution).

For conventional methods different cutoffs have been proposed for the use in PMR and SMR [3] based on data demonstrating worse prognosis at smaller EROA in patients with SMR [25]. For 3D-PISA evaluation different cutoffs may not be necessary, however more data is needed to definitely answer this question. Previous results and our work have shown that 3D-PISA is superior to 2D-PISA especially in SMR and that further improvement can be achieved by replacing a one-time measurement of MR with an integration over the full regurgitation period. The cutoffs we found remain preliminary and the ongoing multicenter POMAR study should establish values on clinical grounds for patients with SMR. Further improvement in the software would be helpful for use of averaged values in routine practice.

Limitations

Although the 3D-PISA as used in this study appears to be superior to it's 2D counterpart, we must be aware of the limitations of color Doppler in detecting the true isovelocity surface. Detected velocities depend on the angle of flow relative to the transducer which represents a theoretical limitation to the method. Furthermore calculation of EROA assumes the PISA and peak velocity to occur simultaneously, which is never true due to limitations in temporal resolution of color Doppler and the technical inability to acquire both Doppler modalities simultaneously. Nevertheless, we were able to demonstrate that EROA determined with 3D color Doppler is useful in clinical practice and averaging over the cardiac cycle further improves results.

\section{Conclusion}

In a diverse population with average ultrasound conditions the use of 3D-PISA and simple calculation of a mean systolic regurgitation orifice area proved to be superior to 2D measures (VC, 2D-PISA, jet area) for distinguishing moderate from severe MR. Use of Real-time non-stitched 3D-Color-Doppler echocardiography with semiautomated PISA analysis is helpful in the evaluation, classification and grading of MR in a routine clinical cardiology setting, where many other methods fail. 3D-PISA reduces assumptions and seemingly improves diagnostic value as compared with 2D-PISA.

Conflict of interest No conflicts of interest were declared by the authors.

\section{References}

1. Bargiggia GS, Tronconi L, Sahn DJ, Recusani F, Raisaro A, De Servi S et al (1991) A new method for quantitation of mitral regurgitation based on color flow Doppler imaging of flow convergence proximal to regurgitant orifice. Circulation 84: $1481-1489$ 
2. Zoghbi WA, Enriquez-Sarano M, Foster E, Grayburn PA, Kraft CD, Levine RA et al (2003) Recommendations for evaluation of the severity of native valvular regurgitation with two-dimensional and Doppler echocardiography. J Am Soc Echocardiogr 16:777-802

3. Lancellotti P, Moura L, Pierard LA (2010) European Association of Echocardiography. European Association of Echocardiography recommendations for the assessment of valvular regurgitation. Part 2: mitral and tricuspid regurgitation (native valve disease). Eur J Echocardiogr 11:307-332

4. Vahanian A, Alfieri O, Andreotti F, Antunes MJ, Baron-Esquivias G, Baumgartner $\mathrm{H}$ et al (2012) Guidelines on the management of valvular heart disease (version 2012): the Joint Task Force on the Management of Valvular Heart Disease of the European Society of Cardiology (ESC) and the European Association for Cardio-Thoracic Surgery (EACTS). Eur Heart J 33:2451-2496

5. Enriquez-Sarano M, Miller FAJ, Hayes SN, Bailey KR, Tajik AJ, Seward JB (1995) Effective mitral regurgitant orifice area: clinical use and pitfalls of the proximal isovelocity surface area method. J Am Coll Cardiol 25:703-709

6. Pu M, Vandervoort PM, Greenberg NL, Powell KA, Griffin BP, Thomas JD (1996) Impact of wall constraint on velocity distribution in proximal flow convergence zone. Implications for color Doppler quantification of mitral regurgitation. J Am Coll Cardiol 27:706-713

7. Iwakura K, Ito H, Kawano S, Okamura A, Kurotobi T, Date M et al (2006) Comparison of orifice area by transthoracic threedimensional Doppler echocardiography versus proximal isovelocity surface area (PISA) method for assessment of mitral regurgitation. Am J Cardiol 97:1630-1637

8. Matsumura Y, Fukuda S, Tran H, Greenberg NL, Agler DA, Wada $\mathrm{N}$ et al (2008) Geometry of the proximal isovelocity surface area in mitral regurgitation by 3-dimensional color Doppler echocardiography: difference between functional mitral regurgitation and prolapse regurgitation. Am Heart J 155:231-238

9. Kahlert P, Plicht B, Schenk IM, Janosi RA, Erbel R, Buck T (2008) Direct assessment of size and shape of noncircular vena contracta area in functional versus organic mitral regurgitation using real-time three-dimensional echocardiography. J Am Soc Echocardiogr 21:912-921

10. Altiok E, Hamada S, van Hall S, Hanenberg M, Dohmen G, Almalla M et al (2011) Comparison of direct planimetry of mitral valve regurgitation orifice area by three-dimensional transesophageal echocardiography to effective regurgitant orifice area obtained by proximal flow convergence method and vena contracta area determined by color Doppler echocardiography. Am J Cardiol 107:452-458

11. Li X, Shiota T, Delabays A, Teien D, Zhou X, Sinclair B et al (1999) Flow convergence flow rates from 3-dimensional reconstruction of color Doppler flow maps for computing transvalvular regurgitant flows without geometric assumptions: an in vitro quantitative flow study. J Am Soc Echocardiogr 12:1035-1044

12. Yosefy C, Levine RA, Solis J, Vaturi M, Handschumacher MD, Hung J (2007) Proximal flow convergence region as assessed by real-time 3-dimensional echocardiography: challenging the hemispheric assumption. J Am Soc Echocardiogr 20:389-396

13. Little SH, Igo SR, Pirat B, McCulloch M, Hartley CJ, Nose Y et al (2007) In vitro validation of real-time three-dimensional color Doppler echocardiography for direct measurement of proximal isovelocity surface area in mitral regurgitation. Am J Cardiol 99:1440-1447

14. Pirat B, Little SH, Igo SR, McCulloch M, Nose Y, Hartley CJ et al (2009) Direct measurement of proximal isovelocity surface area by real-time three-dimensional color Doppler for quantitation of aortic regurgitant volume: an in vitro validation. J Am Soc Echocardiogr 22:306-313

15. Sitges M, Jones M, Shiota T, Qin JX, Tsujino H, Bauer F et al (2003) Real-time three-dimensional color Doppler evaluation of the flow convergence zone for quantification of mitral regurgitation: validation experimental animal study and initial clinical experience. J Am Soc Echocardiogr 16:38-45

16. Grady L, Datta S, Kutter O, Duong C, Wein W, Little SH et al (2011) Regurgitation quantification using 3D PISA in volume echocardiography. Med Image Comput Comput Assist Interv 14:512-519

17. Cobey FC, McInnis JA, Gelfand BJ, Rapo MA, D'Ambra MN (2012) A method for automating 3-dimensional proximal isovelocity surface area measurement. J Cardiothorac Vasc Anesth 26:507-511

18. de Agustin JA, Marcos-Alberca P, Fernandez-Golfin C, Goncalves A, Feltes G, Nunez-Gil IJ et al (2012) Direct measurement of proximal isovelocity surface area by single-beat three-dimensional color Doppler echocardiography in mitral regurgitation: a validation study. J Am Soc Echocardiogr 25:815-823

19. Thavendiranathan P, Liu S, Datta S, Rajagopalan S, Ryan T, Igo SR et al (2013) Quantification of chronic functional mitral regurgitation by automated 3 -d peak and integrated proximal isovelocity surface area and stroke volume techniques using realtime 3-D volume color Doppler echocardiography. In vitro and clinical validation. Circ Cardiovasc Imaging 6:125-133

20. Schwammenthal E, Chen C, Benning F, Block M, Breithardt G, Levine RA (1994) Dynamics of mitral regurgitant flow and orifice area. Physiologic application of the proximal flow convergence method: clinical data and experimental testing. Circulation 90:307-322

21. Buck T, Plicht B, Kahlert P, Schenk IM, Hunold P, Erbel R (2008) Effect of dynamic flow rate and orifice area on mitral regurgitant stroke volume quantification using the proximal isovelocity surface area method. J Am Coll Cardiol 52:767-778

22. Quinones MA, Douglas PS, Foster E, Gorcsan Jr, Lewis JF, Pearlman AS et al (2003) American College of Cardiology/ American Heart Association clinical competence statement on echocardiography: a report of the American College of Cardiology/American Heart Association/American College of Physicians-American Society of Internal Medicine Task Force on Clinical Competence. Circulation 107:1068-1089

23. Buck T, Plicht B (2006) Erbel R [Current recommendations on echocardiographic evaluation of the severity of mitral regurgitation: standardization and practical application using a scoring system]. Herz 31:30-37

24. Thomas L, Foster E, Hoffman JI, Schiller NB (1999) The Mitral Regurgitation Index: an echocardiographic guide to severity. J Am Coll Cardiol 33:2016-2022

25. Lancellotti P, Gerard PL, Pierard LA (2005) Long-term outcome of patients with heart failure and dynamic functional mitral regurgitation. Eur Heart J 26:1528-1532 